\title{
CLASSIFYING SPACES FOR FOLIATIONS WITH ISOLATED SINGULARITIES
}

\author{
PETER GREENBERG
}

\begin{abstract}
Let $\Gamma^{a} \subset \Gamma$ be transitive pseudogroups on $\mathbf{R}^{n}$, such that, for any element $g: U \rightarrow V$ of $\Gamma$, there is a locally finite subset $S \subset U$, such that $\left.g\right|_{U-S}$ is an element of $\Gamma^{a}$. We construct $B \Gamma$, up to weak homotopy type, from $B \Gamma^{a}$ and the classifying spaces of certain groups of germs.

As an application, the classifying space of the pseudogroup of orientation-preserving, piecewise linear homeomorphisms between open subsets of $\mathbf{R}$ is weakly homotopy equivalent to $B \mathbf{R} * B \mathbf{R}$.
\end{abstract}

\section{Introduction.}

1.1 Main result. Let $\Gamma^{a} \subset \Gamma$ be pseudogroups on an $n$-manifold $X$, and let $b \in X$. Assume that the $\Gamma$-orbit orb of $b$ is the same as the $\Gamma^{a}$-orbit of $b$, and that for every $g: U \rightarrow V$ in $\Gamma(U, V$ open in $X)$ there is a locally finite set $S \subset U \cap$ orb such that $\left.g\right|_{U-S} \in \Gamma^{a}$. Let $G$ (respectively $G^{a}$ ) be the discrete group of germs of $\Gamma$-embeddings (respectively, $\Gamma^{a}$-embeddings) from a neighborhood of $b$ to $X$, fixing $b$. In $\S 2$ we copy Tsuboi's construction [Ts] of the $\mathbf{R}^{n}$-microbundles $G \backslash \mathbf{R}^{n}$ and $G^{a} \backslash \mathbf{R}^{n}$ over $B G$ and $B G^{a}$. Removing zero sections, we obtain $\mathbf{R}^{n}-0$ microbundles $G \backslash \mathbf{R}^{n}-0$ and $G^{a} \backslash \mathbf{R}^{n}-0$. Maps are defined to construct a commutative diagram

$$
\begin{array}{ccccc}
G \backslash \mathbf{R}^{n} & & \\
G \backslash \mathbf{R}^{n}-0 & \nearrow & \uparrow & \nwarrow & \\
& \searrow & G^{a} \backslash \mathbf{R}^{n}-0 & \rightarrow & G^{a} \backslash \mathbf{R}^{n} \\
& & \downarrow & \swarrow & \\
& & B \Gamma^{a} & &
\end{array}
$$

Further, there are maps from each space in (1.2) to $B \Gamma$, commuting with the maps in (1.2). Therefore, there is a map from the homotopy colimit of (1.2) to $B \Gamma$.

1.3 THEOREM. The map from the homotopy colimit of (1.2) to $B \Gamma$ is a weak (homotopy) equivalence.

The homotopy colimit [BK, T2] of (1.2) is constructed as follows: let $\Delta^{n}$ be the $n$-simplex. Starting with the disjoint union of the spaces in (1.2), glue in the mapping cylinder of each map. Then attach a copy of $G^{a} \backslash \mathbf{R}^{n}-0 \times \Delta^{2}$ for each of the four commutative triangles in (1.2). The resulting space is homotopy equivalent to the homotopy pushout of $G \backslash \mathbf{R}^{n} \leftarrow P \rightarrow B \Gamma^{a}$, where $P$ is the homotopy pushout of $G \backslash \mathbf{R}^{n}-0 \leftarrow G^{a} \backslash \mathbf{R}^{n}-0 \rightarrow G^{a} \backslash \mathbf{R}^{n}$.

Received by the editors October 27, 1986.

1980 Mathematics Subject Classification (1985 Revision). Primary 58H05; Secondary 55H99. 
1.4 Discussion. It has proven difficult to obtain complete information about the topology of classifying spaces of pseudogroups. However, for a pseudogroup $\Gamma^{a}$ which is the set of restrictions of elements of a group $H$ of analytic diffeomorphisms of an analytic manifold $X$, the classifying space is homotopy equivalent to the homotopy quotient $E H \times{ }_{11} X$ of the action. One might hope that by enlarging the pseudogroup slightly, by adding isolated singularities, one would obtain a pseudogroup $\Gamma$. whose classifying space could be understood; Theorem 1.3 tells how to construct $B \Gamma$ by gluing the classifying space $B G$ of the group of germs at the singular points to $B \Gamma^{a}$.

The main technical difficulty lies in the fact that, a priori, a group of germs does not act on anything larger than a point. Fortunately, a construction of Tsuboi [Ts] builds étale simplicial spaces (that is, simplicial spaces in which all structure maps are étale) which model, up to homotopy, the microbundles asociated to groups of germs, and which, moreover, enjoy (when appropriate) étale simplicial maps to the simplicial spaces whose geometrical realizations, after Haefliger [H1] are the classifying spaces for pseudogroups. Segal's methods [Se3, Appendix] give us sufficient control of the homotopy colimit of (1.2) to prove 1.3.

Formulations of (1.3), for pseudogroups acting on the real line, lead to explicit calculations (1.10).

1.5 COROllary. Let $\Gamma^{a} \subset \Gamma$ be a simple extension, with singularities in the orbit of $b \in \mathbf{R}(2.2)$, of orientation-preserving pseudogroups on $\mathbf{R}$. There are spaces $L_{1}, L_{2}$ neakly equivalent to $B G, B \Gamma^{a}$ respectively, and a commutative diagram

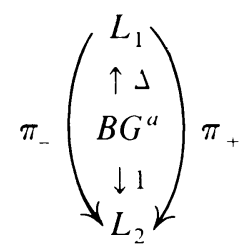

whose homotopy colimit is weakly equivalent to $B \Gamma$.

1.7 Remark. The maps $\pi_{-}$and $\pi_{+}$are "restriction to the left" and "restriction to the right" of a singular germ.

In some instances (1.10) the maps $\pi_{-}, \pi_{+}: B G \rightarrow B \Gamma^{a}$ factor, up to homotopy, through $B G^{a}$, via homomorphisms $p_{B p^{+}}, p_{-}: G \rightarrow G^{a}$. In such cases, let $R$ denote the

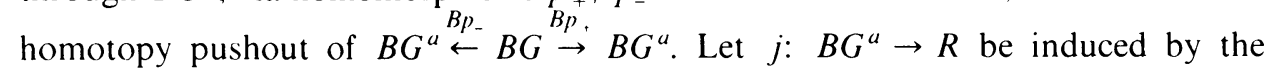
inclusion $B G^{a} \rightarrow B G$, and let $i: B G^{a} \rightarrow B \Gamma$ be the inclusion.

1.8 Corol.t.ary. In the situation just described there are models (up to weak equivalence) of $B \Gamma^{a}, B G^{a}$ and $R$ and a diagram $B \Gamma^{a} \stackrel{i}{\leftarrow} B G^{a} \stackrel{j}{\rightarrow} R$ whose homotopy pushout is weakly equivalent to $B \Gamma$.

The proofs of 1.7 and 1.8 are posponed to $\S 3$.

1.9 BASIC EXAMPLE. The group $\mathbf{Z}$ acts on the line $\mathbf{R}$ as the set of homeomorphisms $g_{n}(x)=2^{n} x, n \in \mathbf{Z}$. Let $\Gamma^{a}$ be the pseudogroup of restrictions of the $g_{n}$ to open subsets of the line. Let $\Gamma$ be the pseudogroup of homeomorphisms $g: U \rightarrow V, U$, 
$V \subseteq \mathbf{R}$ where if $0 \notin U$ then $g \equiv g_{n}$ for some $n \in \mathbf{Z}$, and if $0 \in U$ then

$$
g(x)= \begin{cases}g_{n}(x), & x \leqslant 0 \\ g_{m}(x), & x \geqslant 0\end{cases}
$$

for some $m, n \in \mathbf{Z}$.

We apply 1.8 to $\Gamma^{a} \subset \Gamma$.

Since $G^{a} \simeq \mathbf{Z}, G \simeq \mathbf{Z} \times \mathbf{Z}$ we have $R \approx S^{3}$, so that $B \Gamma$ is weakly equivalent to the pushout $S^{1} \leftarrow S^{1} \rightarrow S^{3}$, that is, to $S^{3}$.

This result can also be obtained from considerations in [H2].

Adding translations to the above $\Gamma$, we obtain more interesting pseudogroups which still have small classifying spaces. For example, let $A$ be the ring $a+b \sqrt{2}, a$, $b \in \mathbf{Z}$. The group of units in $A$ is isomorphic to $\mathbf{Z}$, generated by $u=1+\sqrt{2}$. Let $H$ be the affine group of homeomorphisms of $\mathbf{R}$ of the form $g(x)=u^{n} x+r, n \in \mathbf{Z}$, $r \in A$, and let $\Gamma^{a}$ be the pseudogroup of restrictions of elements of $H$ to open subsets of $\mathbf{R}$. Let $\Gamma$ be the pseudogroup of piecewise-linear homeomorphisms, which on each piece agree with some element of $H$, and which have isolated bendpoints in $A$. We apply 1.8 to the pair $\Gamma^{a} \subset \Gamma$.

Since $G^{a} \simeq \mathbf{Z}, G \simeq \mathbf{Z} \times \mathbf{Z}$ we have $R \approx S^{3}$. Thus $B \Gamma$ is weakly equivalent to the homotopy pushout $B H \leftarrow S^{1} \rightarrow S^{3}$, which is homotopy equivalent to $S^{3} \vee C$, where $C$ is the homotopy cofiber of $S^{1} \rightarrow B H$. It is easy to compute (since $H$ is an extension $\mathbf{Z}^{2} \rightarrow H \rightarrow \mathbf{Z}$ ) that $H_{i} C \simeq \mathbf{Z} / 2, i=1,2$, and $H_{i} C=0, i>2$.

1.10 Piecewise linear homeomorphisms. Let $\Gamma^{a}$ be the pseudogroup of orientationpreserving affine homeomorphisms between open subsets of the real line, and let $\Gamma$ be the pseudogroup of orientation-preserving, piecewise linear homeomorphisms, with isolated "bendpoints", between open subsets of the real line. We apply 1.8.

We have $G^{a} \simeq \mathbf{R}_{+}, G \simeq \mathbf{R}_{+} \times \mathbf{R}_{+}$, where $\mathbf{R}_{+}$is the group of positive real numbers under multiplication. Consequently, $R$ is homotopy equivalent to the join $B \mathbf{R}_{+} * B \mathbf{R}_{+}$, so that $B \Gamma$ is weakly equivalent to the homotopy pushout of $B \Gamma^{a} \leftarrow$ $B \mathbf{R}_{+} \rightarrow B \mathbf{R}_{+} * B \mathbf{R}_{+}$.

As we prove below (Lemma 1.13), $B \mathbf{R}_{+} \rightarrow B \Gamma^{a}$ induces an isomorphism in integral homology. Further, $B \Gamma$ is simply connected (since the topological category whose geometrical realization is $B \Gamma$ has a connected space of morphisms). Hence, $B \mathbf{R}_{+} * B \mathbf{R}_{+} \rightarrow B \Gamma$ is a weak homotopy equivalence, and we can calculate immediately:

1.11 Corollary. $H_{1}(B \Gamma ; \mathbf{Z})=H_{2}(B \Gamma ; \mathbf{Z})=0$, and for $k \geqslant 3$,

$$
H_{k}(B \Gamma ; \mathbf{Z}) \simeq \bigoplus_{i=1}^{k-2} \bigwedge^{i} \mathbf{R}_{+} \otimes \bigwedge^{k-1-i} \mathbf{R}_{+},
$$

where $\wedge^{i} \mathbf{R}_{+}$is the ith exterior product of $\mathbf{R}_{+}$over $\mathbf{Z}$, and where the tensor product is over $\mathbf{Z}$.

Let $G$ be the group of compactly supported piecewise-linear homeomorphisms of R. Epstein [E, 3.2] showed that $G$ is simple, and hence that $H_{1}(G ; \mathbf{Z})=0$. We can compute a little further by applying Mather's theorem [Ma] that there is a map $B G \rightarrow \Omega B \Gamma$ which induces an isomorphism in homology. 
1.12 Corollary. $H_{2}(G ; \mathbf{Z})=\mathbf{R}_{+} \otimes \mathbf{R}_{+}$, and $H_{3}(G ; \mathbf{Z})=\left(\bigwedge^{2} \mathbf{R}_{+} \otimes \mathbf{R}_{+}\right) \oplus\left(\mathbf{R}_{+}\right.$ $\left.\otimes \wedge^{2} \mathbf{R}_{+}\right)$.

Proof. [Ma, Corollary 3.5].

Finally, we prove

1.13 Lemma. $B \mathbf{R}_{+} \rightarrow B \Gamma^{a}$ induces an isomorphism in integral homology.

Proof. $B \Gamma^{a}$ is homotopy equivalent to the classifying space of the discrete group Aff of orientation preserving affine homeomorphisms of the real line. We apply the "center kills" technique of Sah [Sa] to prove that the homomorphism $\mathbf{R}_{+} \rightarrow$ Aff induces an isomorphism in homology.

Considering the Leray-Serre spectral sequence of the exact sequence $\mathbf{R} \rightarrow$ Aff $\rightarrow$ $\mathbf{R}_{+}$, it suffices to prove that $H_{p}\left(\mathbf{R}_{+} ; H_{q} \mathbf{R}\right)=0, q>0$. To begin, if $r \in \mathbf{R}_{+}$, the map $r: \mathbf{R} \rightarrow \mathbf{R}$ induces $r_{q}: H_{q}(\mathbf{R}) \rightarrow H_{q}(\mathbf{R})$, and hence an isomorphism $\left(r_{q}\right)_{*}$ of $H_{*}\left(\mathbf{R}_{+} ; H_{q} \mathbf{R}\right)$. But $\left(r_{q}\right)_{*}$ is the same as the map in homology induced by conjugation by $r$ in the group $\mathbf{R}_{+}$, so that $\left(r_{q}\right)_{*}$ is the identity.

Recall that $H_{q} \mathbf{R}=\wedge^{q} \mathbf{R}$, the $q$ th exterior product of $\mathbf{R}$ over $\mathbf{Z}$, and that $r_{q}$ is given on generators by $r_{q}\left(s_{1} \wedge \cdots \wedge s_{q}\right)=r s_{1} \wedge \cdots \wedge r s_{q}$. In particular, 2 -id is multiplication by $2^{q}-1$, which is an isomorphism for $q>0$ because $H_{q} \mathbf{R}$ is torsion free. Hence $\left(2_{q}-\mathrm{id}\right)_{*}=\left(2_{q}\right)_{*}-\mathrm{id}_{*}=\mathrm{id}-\mathrm{id}=0$ is an isomorphism of $H_{*}\left(\mathbf{R}_{+} ; H_{q} \mathbf{R}\right), q>0$, which must therefore be 0 .

1.14 AcKNOWLEDGMENTS. I would like to thank Sol Jekel and Vlad Sergiescu for helpful discussion and correspondence, and the referee for careful reading and valuable suggestions.

1.15 Organization. $\$ 2$ reformulates and proves the main theorem. $\$ 3$ proves Corollaries 1.5 and 1.8. $\S 4$ gives a geometric interpretation of (1.3).

1.16 Notation. We freely use simplicial notation. References are [M, Se1].

Recall [Se1] that a topological category is a small category, in which the sets of objects and morphisms have topologies so that the structure maps are continuous. A (topological) category with a (continuous) inverse is called a (topological) groupoid.

The nerve of a topological category $C$ is a simplicial space $C_{*}$; a continuous functor $F: C \rightarrow D$ induces a simplicial map $F_{*}: C_{*} \rightarrow D_{*}$. If $C$ is a topological category we denote by $R, D: C_{n} \rightarrow C_{0}$ the domain and range maps $D=d_{0} \circ \cdots \circ d_{n}$, $R=d_{n-1} \circ \cdots \circ d_{0}$.

We employ the two geometric realization functors $|\cdot|$ and $\|\cdot\|$ discussed by Segal [Se2]. If $A_{*}$ is a simplicial space, $\left\|A_{*}\right\|$ is defined by gluing together the $A_{n} \times \Delta^{n}$ by setting $\left(F a_{n}, t_{k}\right) \sim\left(a_{n}, F^{*} t_{k}\right)$ for every composite of face maps $F: A_{n} \rightarrow A_{k} ; F^{*}$ : $\Delta^{k} \rightarrow \Delta^{n}$ is the induced inclusion. If we further set $\left(S a_{n}, t_{k}\right) \sim\left(a_{n}, S^{*} t_{k}\right)$ for every composite $S$ of degeneracy maps we obtain $\left|A_{*}\right|$.

The realization functor $\|\cdot\|$ is useful to us because of the following:

1.17 Proposition [Se2, APpendix A.1, ii]. If $f_{*}: A_{n} \rightarrow B_{n}$ is a map of simplicial spaces so that each $f_{n}: A_{n} \rightarrow B_{n}$ is a weak equivalence, then $\left\|f_{*}\right\|:\left\|A_{*}\right\| \rightarrow B_{*} \|$ is a weak equivalence. 
Homotopy colimits are defined as in [BK or T2]. We shall use the following consequence of [T2], (3.5) and (3.11) on multiple homotopy colimits.

1.18 Proposition. Let $D_{*}$ be a diagram of simplicial spaces. Denote by $D_{k}$ the diagram of the spaces of $k$-simplices of the simplicial spaces in $D_{*}$, by $\left\|D_{*}\right\|$ the diagram of the geometric realizations of the simplicial spaces in $D_{*}$, and by hocolim $D_{k}$ the simplicial space whose space of $k$-simplices is the homotopy colimit of $D_{k}$.

There is a weak homotopy equivalence from $\|$ hocolim $D_{k} \|$ to the homotopy colimit of $\left\|D_{*}\right\|$.

2. A model for $B \Gamma$. We make the definitions necessary for a precise statement of the main theorem.

2.1 Theorem. A groupoid $\Gamma$ étale over its objects $X$ (or a geo $\Gamma$ on $X$ ) is a topological groupoid $\Gamma$, whose space of objects is $X$, such that the domain and range maps $D, R: \Gamma_{1} \rightarrow X$ are locally homeomorphisms.

As described, for example, in [St] one can associate to any pseudogroup $\Gamma$ on $X$ a geo on $X$ which we also call $\Gamma$. The space of morphisms of the geo $\Gamma$ is the sheaf of germs of elements of the pseudogroup $\Gamma$. Further, the geometric realization $\|\Gamma\|$ of the geo is a standard model for the classifying space $B \Gamma$.

A geo is a more general object than a pseudogroup; consider the following example. Let $\Gamma^{2}$ be the geo associated to the pseudogroup of all homeomorphisms between open subsets of $\mathbf{R}^{2}$, and let $\Gamma^{2,1}$ be the full subcategory of $\Gamma^{2}$ whose space of objects is $\mathbf{R}$, considered as a line in $\mathbf{R}^{2}$. Essentially, $\Gamma^{2,1}$ is the geo associated to the "pseudogroup" whose elements are homeomorphisms between open subset of $\mathbf{R}$, with germs of extensions to a neighborhood in $\mathbf{R}^{2}$.

2.2 Definition. Let $\Gamma^{a} \subseteq \Gamma$ be geos on a (topological) $n$-dimensional manifold $X$, and let $b \in X$. We say that $\Gamma^{a} \subseteq \Gamma$ is a simple extension with singularities in the orbit of $b$ provided

(i) $\left\{\operatorname{Rg}(b): g \in \Gamma_{1}^{a}, D g=b\right\}=\left\{\operatorname{Rg}(b): g \in \Gamma_{1}, D g=b\right\}$,

(ii) if $U$ is an open subset of $X$, and $g: U \rightarrow \Gamma_{1}$ is a section of the domain map, then there is a locally finite subset $S$ of $U \cap\left\{\operatorname{Rg}(b) ; g \in \Gamma_{1}, D g=b\right\}$ such that $g(U-S) \subseteq \Gamma_{1}^{a}$.

For the rest of $\S 2$, let $\Gamma^{a} \subset \Gamma$ be a simple extension with singularities in the orbit of $b$.

2.3 Definition. Let $\underline{G}$ be the discrete groupoid with objects $\underline{G}_{0}=\{\operatorname{Rg}(b) ; g \in$ $\left.\Gamma_{1}, D g=b\right\}$ and morphisms $\underline{G}_{1}=\left\{g \in \Gamma_{1}: D g \in \underline{G}_{0}\right\}$, and structure maps induced from $\Gamma$. Let $\underline{G}^{a}$ be the subcategory of $\underline{G}$ with the same set of objects, and morphisms $\underline{G}_{1}^{a}=\underline{G}_{1} \cap \Gamma_{1}^{a}$.

If $\Gamma^{a}$ and $\Gamma$ act transitively on $X$, then $\underline{G}^{a}$ and $\underline{G}$ are $\Gamma^{a}$ and $\Gamma$, respectively, but with the discrete topology. In any case, if $\Gamma$ is the geo associated to a pseudogroup then the realizations $\left\|\underline{G}^{a}\right\|$ and $\|\underline{G}\|$ are homotopy equivalent to the classifying space $B G^{a}$ and $B G$ of the groups of germs at $b$.

2.4 Models for the microbundles. The spaces $G \backslash \mathbf{R}^{n}, G^{a} \backslash \mathbf{R}^{n}, G \backslash \mathbf{R}^{n}-0$ and $G^{a} \backslash \mathbf{R}^{n}-0$ are the geometrical realizations of simplicial spaces which we now define, as in [Ts]. We let $d_{i}$ and $s_{j}$ be the face and degeneracy maps both of $\Gamma_{*}$ and $\underline{G}_{*}$. 
2.5 Lemma. There exist, for all $k \geqslant 0$ and $g \in \underline{G}_{k}$, open subsets $U_{g} \subseteq \Gamma_{k}$, homeomorphic to $\mathbf{R}^{n}$, such that

(i) $g \in U_{g}$,

(ii) $U_{g} \cap\left(\Gamma_{k}-\Gamma_{k}^{a}\right) \subseteq\{g\}$,

(iii) $d_{i} U_{g} \subseteq U_{d, g}$ and $d_{i}: U_{g} \rightarrow U_{d_{1} g}$ is a homeomorphism, onto its image, for all $i$,

(iv) $s_{j} U_{g}=U_{s, g}$ and $s_{j}: U_{g} \rightarrow U_{s, g}$ is a homeomorphism, for all $j$.

We postpone the proof of 2.5 until the end of $\S 2$, and assume the existence of the open sets $U_{g}$.

2.6 Definition. Let $C_{*}$ be the simplicial space with $C_{k}=\amalg U_{g}, g \in \underline{G}_{k}$, and structure maps $d_{i}: U_{g} \rightarrow U_{d_{i}}, s_{j}: U_{g} \rightarrow U_{s, g}$ induced from $\Gamma_{*}$. Let $I_{*}: C_{*} \rightarrow \Gamma_{*}$ be the étale simplicial map which is inclusion on each $U_{g}$.

Let $D_{g}=\amalg\left(U_{g}-\{g\}\right), g \in \underline{G}_{k}$, and note that the face and degeneracy maps between the $C_{k}$ restrict to the $D_{k}$, making $D_{*}$ a subsimplicial space of $C_{*}$. Let $J_{*}$ : $D_{*} \rightarrow \Gamma_{*}$ be the composition of the inclusion $D_{*} \rightarrow C_{*}$ with $I_{*}$.

Similarly, $C_{k}^{a}=\amalg U_{g}, g \in \underline{G}_{k}^{a}$, and $D_{k}^{a}=\amalg\left(U_{g}-\{g\}\right), g \in G_{k}^{a}$, are subsimplicial spaces of $C_{k}$, and we define $I_{*}^{a}: C_{*}^{a} \rightarrow \Gamma_{*}, J_{*}^{a}: D_{*}^{a} \rightarrow \Gamma_{*}$ be the étale simplicial maps which are the restriction of $I_{*}$ to $C_{*}{ }^{a}$ and $D_{*}^{a}$.

2.7 Remark. There is a simplicial map $p_{*}: C_{*} \rightarrow \underline{G}_{*}$, defined by setting $p_{k}(x)=g$ if $x \in U_{g}$, and $p_{*}$ has a section $\sigma_{*}: \underline{G}_{*} \rightarrow C_{*}$ defined by $\sigma_{k}(g)=g \in U_{g}$. By (1.17) $\left\|p_{*}\right\|:\left\|C_{*}\right\| \rightarrow\left\|\underline{G}_{*}\right\|$ is a homotopy equivalence, since each $U_{g}$ is contractible. Further, $C_{k}-\sigma_{k}\left(\underline{G}_{k}\right)=D_{k}$. With this motivation, we define $G \backslash \mathbf{R}^{n}=\left\|C_{*}\right\|, G \backslash \mathbf{R}^{n}$ - $0=\left\|D_{*}\right\|, G^{a} \backslash \mathbf{R}^{n}=\left\|C_{*}^{a}\right\|, G^{a} \backslash \mathbf{R}^{n}-0=\left\|D_{*}^{a}\right\|$.

The maps $J_{k}, I_{k}^{a}$ and $J_{k}^{a}$ factor through the inclusion $\Gamma_{k}^{a} \rightarrow \Gamma_{k}$, giving a diagram

$$
\begin{array}{cccccc} 
& & C_{k} & & & \\
& \nearrow & \uparrow & \nwarrow & \\
D_{k} & \leftarrow & D_{k}^{a} & \rightarrow & C_{k}^{a} \\
& \searrow & \downarrow & \swarrow & \\
& & \Gamma_{k}^{a} & &
\end{array}
$$

of simplicial spaces and étale simplicial maps (the maps on the upper half of (2.8) ${ }_{k}$ are inclusions). The $I_{k}, J_{k}, I_{k}^{a}$ and the inclusions $\Gamma_{k}^{a} \rightarrow \Gamma_{k}$ commute, whenever appropriate, with the maps in $(2.8)_{k}$, and therefore glue together to produce a map $F$ from the homotopy colimit of the diagram

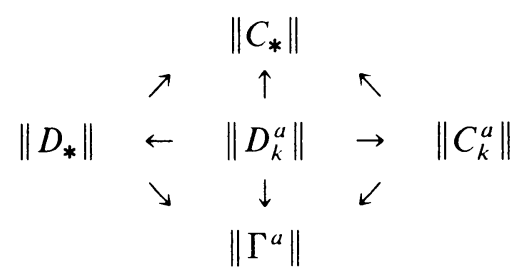

to $\|\Gamma\|$. We now reformulate (and prove) 1.3.

2.10 THEOREM. $F$ is a weak equivalence.

Proof. Let $E_{k}$ be the homotopy colimit of $(2.8)_{k}$, and let $F_{*}: E_{*} \rightarrow \Gamma_{*}$ be the simplicial map built from the $I_{*}, J_{*}, I_{*}^{a}, J_{*}^{a}$ and the inclusion $\Gamma_{*}^{a} \rightarrow \Gamma_{*}$. By 1.18 it 
suffices to prove that each $F_{k}: E_{k} \rightarrow \Gamma_{k}$ is a weak equivalence. By definition of the $U_{g}$ (2.5)(i) $F_{k}$ is onto, and $E_{k}$ is the homotopy colimit of a diagram of spaces étale over $\Gamma_{k}$. Invoking [Se2, A.6] it is enough to show that the fibers of $F_{k}$ are contractible.

If $g \in \Gamma_{k}-\Gamma_{k}^{a}$, then $g \in U_{h}$ only if $g=h$, so $g \notin D_{k}, g \notin C_{k}^{a}, g \notin D_{k}^{a}$. Hence the fiber $F_{k}^{-1}(g)$ is a single point, and is therefore contractible. Now suppose that $g \in \Gamma_{k}^{a}$.

Consider the top half of diagram $(2.8)_{k}$

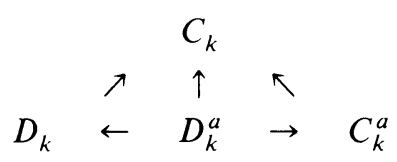

Let $L_{K}$ be the homotopy colimit of $(2.11)_{k}$, and let $f_{k}: L_{k} \rightarrow \Gamma_{k}$ be the restriction of $F_{k}$ to $L_{k}$. Since the maps in (2.11) $)_{k}$ are inclusions, the fiber $f_{k}^{-1}(g), g \in \Gamma_{k}$, is a disjoint union of components, with one component $m_{h}$ containing each element $h$ of $I_{k}^{-1}(g)$.

Further if $g \in \Gamma_{k}^{a}$ and $h \in I_{k}^{-1}(g)$ then $h \in C_{k}^{a} \cup D_{k}$. Therefore, if $g \in \Gamma_{k}^{a}$, $F_{k}^{-1}(g)$ is the union of pieces which intersect pairwise in the point $g \in \Gamma_{k}^{a}$, with one piece $M_{h}$ for every element $h$ of $I_{k}^{-1}(g)$. We now prove that each $M_{h}$ is contractible. There are three cases to consider.

(i) $h=g \in U_{g}$. Then $h \notin D_{k}, h \notin D_{k}^{a}$, but since $g \in \Gamma_{k}^{a}, h \in C_{k}^{a}$. Therefore $M_{h}$ is the homotopy colimit of the diagram $\leftarrow \leftarrow \cdot \rightarrow \cdot$, and is contractible.

(ii) $h=g \in U_{\gamma}, g \neq \gamma, \gamma \in \Gamma_{k}^{a}$. Since $\gamma \in \Gamma_{k}^{a}$ and $h \neq \gamma, h \in D_{k}, D_{k}^{a}$ and so $M_{k}$ is the homotopy colimit of the diagram

which is contractible.

(iii) $h=g \in U_{\gamma}, g \neq \gamma, \gamma \in \Gamma_{k}-\Gamma_{k}^{a}$. Since $h \in U_{\gamma}-\{\gamma\}, h \in D_{k}$. But $\gamma \in \Gamma_{k}$ - $\Gamma_{k}^{a}$ so $h \notin C_{k}^{a}$. Thus $M_{h}$ is the homotopy colimit of the diagram $\cdot \leftarrow \cdot \rightarrow \cdot$ and is contractible.

Since the $M_{h}$ are all contractible, $F_{k}^{-1}(g)$ is contractible, so that $F_{k}$ is a weak equivalence.

Proof of Lemma 2.5. Recall [BK] that the simplicial set $\underline{G}_{*}$ is filtered by skeleta $\underline{G}_{*}^{N}$, where $\underline{G}_{k}^{N}=\underline{G}_{k}$ if $k \leqslant N$, and if $k>N, \underline{G}_{k}^{N}=\left\{s(h): h \in \underline{G}_{N}, s: \underline{G}_{N} \rightarrow \underline{G}_{k}\right.$ a composition of degeneracy maps $\}$. We define the $U_{g}, g \in \underline{G}_{*}^{N}$, and show that 2.5(i)-(iv) are satisfied, by induction on $N$.

If $g \in G_{0}=\left\{R h(b): h \in \Gamma_{1}, D h=b\right\}$ let $U_{g}$ be an open subset of $X$ which contains $g$ and is homeomorphic to $\mathbf{R}^{n}$. Such a $U_{g}$ exists because $X$ is an $n$-manifold. If $g \in \underline{G}_{k}^{0}$, then $g=s(h)$, with $h \in \underline{G}_{0}$ and $s$ some composition of degeneracy 
maps. Define $U_{g}=s\left(U_{k}\right)$, where we consider $s$ as the map $s: X \rightarrow \Gamma_{k}$. Clearly $U_{g}$ satisfy (i)-(iv).

Suppose that the $U_{g}$ have been defined so as to satisfy (i)-(iv), for $g \in \underline{U}_{*}^{N-1}$. For each $g \in \underline{G}_{N}^{N}-\underline{G}_{N}^{N-1}$, let $V_{g}=\bigcap_{i=1}^{N} d_{i}^{-1}\left(U_{d, g}\right)$. Because $\Gamma_{N}$ is an $n$-manifold and the $d_{i}$ are locally homeomorphisms, there is an open subset $U_{g}$ of $V_{g}$, containing $g$, homeomorphic to $\mathbf{R}^{n}$, so that $U_{g} \cap\left(\Gamma_{N}-\Gamma_{N}^{a}\right) \subseteq\{g\}$, and so that $d_{i}: U_{g} \rightarrow U_{d_{t} g}$ is one-to-one. These $U_{g}$, for $g \in \underline{G}_{N}^{N}$, satisfy (i)-(iv).

If $g \in \underline{G}_{k}^{N}-\underline{G}_{k}^{N-1}, k>N$, then there is a unique $h \in \underline{G}_{N}^{N}-\underline{G}_{N}^{N-1}$, and some composition of degeneracy maps $s: \underline{G}_{N} \rightarrow \underline{G}_{k}$ so that $g=s h$. Let $U_{g}=s U_{h}$. Certainly (i), (ii) hold; we verify (iii), (iv).

For (iv), suppose that $g \in \underline{G}_{k}^{N}-\underline{G}_{k}^{N-1}$. We want to prove that $s_{i}: U_{g} \rightarrow \Gamma_{k+1}$ is a homeomorphism onto $U_{s, g}$. Write $g=s h$, with $h \in \underline{G}_{N}^{N}$ and $s: \underline{G}_{N} \rightarrow \underline{G}_{k}$ a composition of degeneracy maps. Then $s: U_{h} \rightarrow U_{g}$ is a homeomorphism by definition, as is $s_{i} s: U_{h} \rightarrow U_{s, g}$. Therefore, $s_{i}=\left(s_{i} s\right) s^{-1}: U_{g} \rightarrow U_{s, g}$ is a homeomorphism.

To prove (iii), let $g \in \underline{G}_{k}^{N}-G_{k}^{N-1}$; we show that $d_{i}: U_{g} \rightarrow \Gamma_{k-1}$ is one-to-one, and that $d_{i} U_{g} \subseteq U_{d, g}$. Write $g=s h$ as above. Either there is some composition of degeneracy maps $s^{\prime}$, and some $j$ so that $d_{i} s=s^{\prime} d_{j}$, or else $d_{i} s=s^{\prime}$. Therefore we can write $d_{i} s=s^{\prime} d^{\prime}$, where $d^{\prime}$ is either the identity or a face map. In either case, $d^{\prime}$ : $U_{h} \rightarrow U_{d^{\prime} h}$ is one-to-one, and $s: U_{h} \rightarrow U_{g}$ is by definition a homeomorphism, so $d_{i}=s^{\prime} d^{\prime} s^{-1}: U_{g} \rightarrow U_{d_{i} g}$ is one-to-one.

3. Proofs of $\mathbf{1 . 5}$ and 1.8. The proofs of 1.5 and 1.8 involve the homotopy colimits of certain diagrams. Recall [BK, T2] that the homotopy colimit of a diagram of topological spaces is a space constructed as follows. To the disjoint union of the spaces in the diagram, attach the mapping cylinders of the maps in the diagram; that is, attach a copy of $A \times \Delta^{1}$ for every map $A \stackrel{f}{\rightarrow} B$ in the diagram. Then attach, in a similar way, a copy of $A \times \Delta^{2}$ for every composition $A \stackrel{f}{\rightarrow} B \stackrel{g}{\rightarrow} C$ of maps, and so on.

Note that if the underlying graph of the diagram is finite and has no cycles, the process described above terminates after a finite number of attachments.

3.1 Preliminaries. Consider the homotopy pushout $P$ of $G \backslash \mathbf{R}-0 \leftarrow G^{a} \backslash \mathbf{R}-0$ $\rightarrow G^{a} \backslash \mathbf{R}$. Let $\mathbf{R}_{+}=\{r \in \mathbf{R}: r>0\}$, and let $\mathbf{R}_{-}=\{r \in \mathbf{R}: r<0\}$. From our construction of $G \backslash \mathbf{R}-0$ in $\S 2$, and because $\Gamma$ preserves orientation, it follows that $G \backslash \mathbf{R}-0$ is the disjoint union of spaces $G \backslash \mathbf{R}_{+}$and $G \backslash \mathbf{R}_{-}$, and, likewise, that $G^{a} \backslash \mathbf{R}-0=G^{a} \backslash \mathbf{R}_{+} U G^{a} \backslash \mathbf{R}_{\text {. }}$. We can therefore regard $P$ as the homotopy colimit of the diagram

$$
G \backslash \mathbf{R}_{-} \leftarrow G^{a} \backslash \mathbf{R}_{-} \rightarrow G^{a} \backslash \mathbf{R} \leftarrow G^{a} \backslash \mathbf{R}_{+} \rightarrow G \backslash \mathbf{R}_{+}
$$

and we reformulate Theorem 1.3 as follows.

3.1. COROLlLARY. Let $\Gamma^{a} \subseteq \Gamma$ be a simple extension, with singularities in the orbit of $b \in \mathbf{R}$. of orientation-preserving geos on $\mathbf{R}$. Then there is a weak equivalence from the 
homotopy colimit of the diagram

to $B \Gamma$.

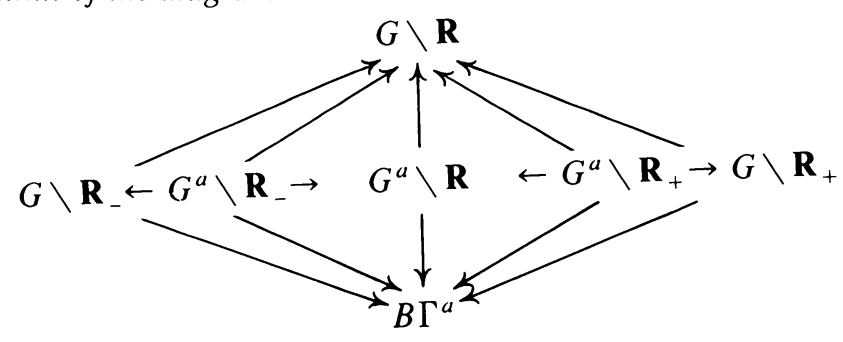

Proof OF 1.5. Let $L_{1}$ be the mapping cylinder of the inclusion $G^{a} \backslash \mathbf{R} \rightarrow G \backslash \mathbf{R}$, and let $L_{2}$ be the homotopy colimit of the diagram

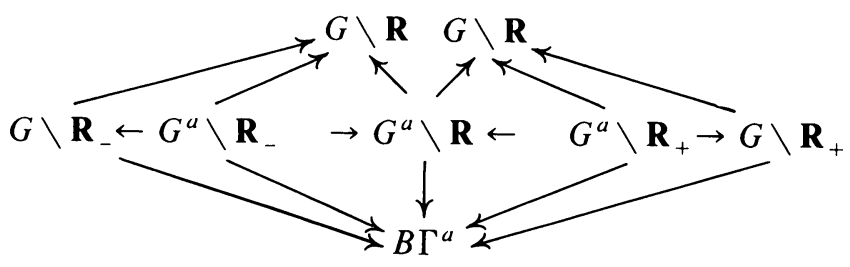

There is a commuting diagram

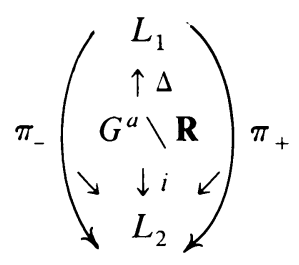

where $\Delta, i, \pi_{-}$and $\pi_{+}$are the obvious inclusions. $L_{1}$ is homotopy equivalent to $G \backslash \mathbf{R}$ and hence to $B G$, so 1.5 follows from the following lemmas.

3.6 LeMmA. $B \Gamma^{a} \rightarrow L_{2}$ is a weak equivalence.

3.7 Lemma. The homotopy colimit of (3.5) is weakly equivalent to $B \Gamma$.

Proof OF 3.6. Let $L_{3}$ be the mapping cylinder of $G^{a} \backslash \mathbf{R} \rightarrow B \Gamma^{a}$, and let $L_{4}$ and $L_{5}$ be the homotopy colimits of
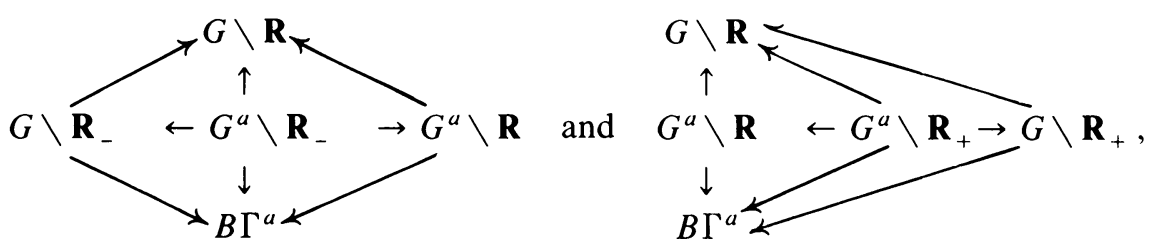

respectively. Then $L_{2}$ is the pushout of $L_{4} \leftarrow L_{3} \rightarrow L_{5}$. Since $G^{a} \backslash \mathbf{R}_{+} \rightarrow G^{a} \backslash \mathbf{R}$ and $G \backslash \mathbf{R}_{+} \rightarrow G \backslash \mathbf{R}$ are weak equivalences, it is not hard to show that $B \Gamma^{a} \rightarrow L_{i}$, $i=3,4,5$, are weak equivalences, whence $B \Gamma^{a} \rightarrow L_{2}$ is a weak equivalence. 
PROOF OF 3.7. Let $K_{2}$ be the homotopy pushout of $G \backslash \mathbf{R} \leftarrow G^{a} \backslash \mathbf{R} \rightarrow G \backslash \mathbf{R}$. This latter is a subdiagram of (3.4), and $\pi_{-}, i$ and $\pi_{+}$factor through $K_{2}$. Letting $K_{3}$ be the homotopy colimit of the diagram

$$
\pi_{-}\left(\begin{array}{c}
L_{1} \\
\uparrow\lrcorner \\
G^{a} \backslash \mathbf{R} \\
\downarrow i \\
K_{2} \downarrow
\end{array}\right) \pi_{+}
$$

we see that the homotopy colimit of (3.5) is the pushout of $L_{2} \leftarrow K_{2} \rightarrow K_{3}$. But $K_{3}$ is homeomorphic to the homotopy colimit of

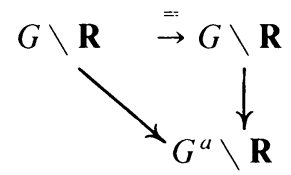

so the pushout of $L_{2} \leftarrow K_{2} \rightarrow K_{3}$ is weakly equivalent to the homotopy colimit of (3.3).

Proof of 1.8. The proof of 1.8 requires that the homomorphisms $p_{+}, p_{-}: G \rightarrow G^{a}$ induce maps $p_{+} \backslash \mathbf{R}_{+}: G \backslash \mathbf{R}_{+} \rightarrow G^{a} \backslash \mathbf{R}_{+}$and $p_{-} \backslash \mathbf{R}_{-}: G \backslash \mathbf{R}_{-} \rightarrow G^{a} \backslash \mathbf{R}_{-}$. We begin, therefore, by slightly altering the open sets $U_{g}$ used in the construction of the simplicial spaces $C_{*}$ and $C_{*}^{a}$. Namely, for $g \in \underline{G}_{k}^{a}$ define the $U_{g}$ following the proof of Lemma 2.5. Then, for $g \in \underline{G}_{k}-\underline{G}_{k}^{a}$, let $U_{g}=\left\{h \in U_{p . g}: D h<D g\right\} \cup\{g\} \cup$ $\left\{h \in U_{p . g}: D h>D g\right\}$. The $U_{g}$ still satisfy $2.5(\mathrm{i})-(\mathrm{iv})$, and the maps $p_{+} \backslash \mathbf{R}_{+}$, $p_{-} \backslash \mathbf{R}_{-}$are well defined.

Without changing the homotopy type of the homotopy colimit, replace (3.3) with the diagram

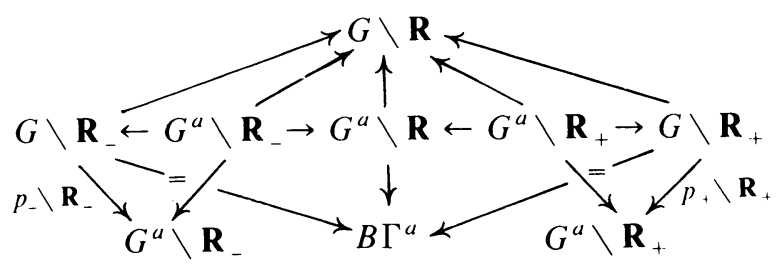

Let $L_{1}$ be the homotopy colimit of the subdiagram

$$
\begin{aligned}
G \backslash \mathbf{R}_{-} \leftarrow G^{a} \backslash \mathbf{R}_{-} \rightarrow G^{a} \backslash \mathbf{R} \leftarrow G^{a} \backslash \mathbf{R}_{+} & \rightarrow G \backslash \mathbf{R}_{+} \\
p_{-} \backslash \mathbf{R}_{-} \searrow \swarrow= & =\searrow \swarrow p+\backslash \mathbf{R}_{+} \\
G^{a} \backslash \mathbf{R}_{-} & G^{a} \backslash \mathbf{R}_{+}
\end{aligned}
$$

and let $L_{2}$ be the homotopy colimit of the subdiagram

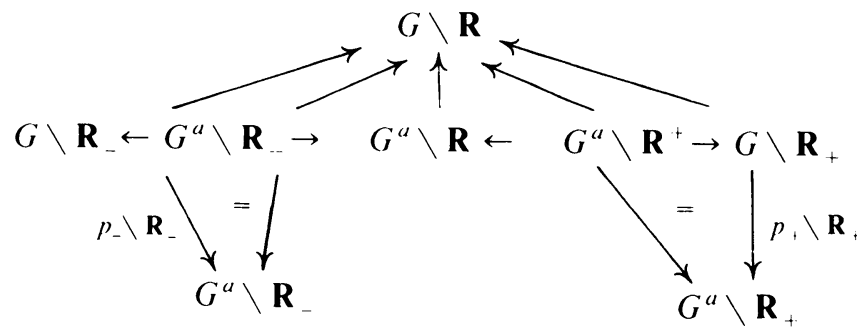


Let $j: L_{1} \rightarrow L_{2}$ be inclusion, and let $i: L_{1} \rightarrow B \Gamma^{a}$ be the map induced by the maps, in (3.8), from the spaces in $L_{1}$ to $B \Gamma^{a}$. There is a diagram $B \Gamma^{a} \stackrel{i}{\leftarrow} L_{1} \stackrel{j}{\rightarrow} L_{2}$ whose pushout is homotopy equivalent to the homotopy colimit of (3.8), and hence weakly equivalent to $B \Gamma$. Clearly, $L_{1}$ is weakly equivalent to $B G^{a}$, and $L_{2}$ retracts to the homotopy colimit of the diagram

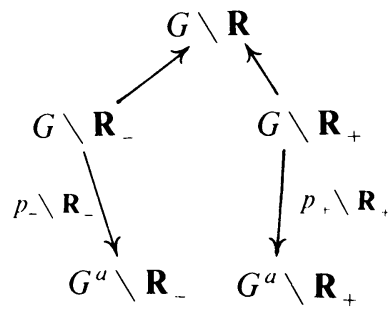

which is weakly equivalent to $R$.

4 Geometrical interpretation. Let $\Gamma^{a} \subset \Gamma$ be pseudogroups on $\mathbf{R}^{n}$, and suppose that $\Gamma^{a} \subset \Gamma$ is a simple extension with singularities in the orbit of $b \in \mathbf{R}^{n}$. Let $M$ be a compact oriented $k$-manifold with a codimension $n \Gamma$-foliation (as described, for example, by charts; see below). We sketch the construction of the classifying map from $M$ to homotopy pushout of $B \Gamma^{a} \leftarrow P \rightarrow G \backslash \mathbf{R}^{n}$. Considering $[M] \in H_{k} B \Gamma$, we describe the image $R_{k}[M] \in H_{k-1} P$ of the connecting map $R_{k}: H_{k} B \Gamma \rightarrow H_{k-1} P$ arising from the Mayer-Vietoris sequence.

There is a (nonunique) subset $S$ of $M$, compact and contained in finitely many leaves, such that the restriction to $M-S$ of the classifying map $M \rightarrow B \Gamma$ factors, up to homotopy, through $B \Gamma^{a}$. (One may construct $S$ as follows: suppose that the foliation $F$ is defined via a finite open cover $\left\{W_{i}\right\}$ of $M$, with submersions $\psi_{i}$ : $W_{i} \rightarrow \mathbf{R}^{\prime \prime}$, such that the fibers $\psi_{i}^{-1}(x)$ are leaves of $F \mid W_{i}$, and such that, where defined, the $\psi_{i} \psi_{j}^{-1}: \psi_{j}\left(W_{i} \cap W_{j}\right) \rightarrow \psi_{i}\left(W_{i} \cap W_{j}\right)$ are elements of $\Gamma$. Let $S_{i j} \subset$ $\psi_{j}\left(W_{i} \cap W_{j}\right)$ be the singular set of $\psi_{i} \psi_{j}^{-1}$. We can assume, shrinking the $W_{i}$ slightly if necessary, that the $S_{i j}$ are finite sets. Now set $S=\cup_{i, j} \psi_{j}^{-1}\left(S_{i, j}\right)$.) However $S$ is defined, we can assume that $S$ is the union $S=\bigcup_{j=1}^{m} K_{j} \bigcup_{i=1}^{\prime} N_{i}$ where each $K_{i}$ is a compact leaf and each $N_{j}$ is a compact $(k-n)$-submanifold with boundary, of a possibly noncompact leaf $L_{j}$.

Let $U_{i}, 1 \leqslant i \leqslant l$, and $V_{j}, 1 \leqslant j \leqslant m$, be nonintersecting open neighborhoods which retract to the $N_{i}$ and $K_{j}$, respectively. $M$ is covered by the two open sets $\cup U_{i} \cup V_{j}$ and $M-S$, which intersect in $\cup\left(U_{i}-N_{i}\right) \cup\left(V_{j}-K_{j}\right)$. We describe the map from $M$ to the homotopy pushout of $B \Gamma^{a} \leftarrow P \rightarrow G \backslash \mathbf{R}^{n}$ by giving maps $A$ : $\cup U_{i} \cup V_{j} \rightarrow G \backslash \mathbf{R}^{n}, B: M-S \rightarrow B \Gamma^{a}$ and a compatible $C: \cup\left(U_{i}-N_{i}\right) \cup\left(V_{j}-K_{j}\right) \rightarrow P$.

The retractions of the $U_{i}$ and the $V_{j}$ to the $N_{i}$ and $K_{j}$, respectively, and the holonomy microbundles over the $N_{i}$ and $K_{j}$, define the map $A$. As we observed, $S$ is defined so that $M-S \rightarrow B \Gamma$ factors, up to homotopy, through $B \Gamma^{a}$. Let $B$ : $M-S \rightarrow B \Gamma^{a}$ be such a factoring.

We describe $C$ : $\cup\left(U_{i}-N_{i}\right) \cup\left(V_{j}-K_{j}\right) \rightarrow P$ by describing the restrictions $C_{i}$ : $U_{i}-N_{i} \rightarrow P$ and $C_{j}: V_{j}-K_{j} \rightarrow P$. Recall that $P$ is the homotopy pushout of $G \backslash \mathbf{R}^{n}-0 \leftarrow G^{a} \backslash \mathbf{R}^{n}-0 \rightarrow G^{a} \backslash \mathbf{R}^{n}$. The map is the composition $C_{j}: V_{j}-K_{j} \rightarrow$ $G \backslash \mathbf{R}^{n}-0 \rightarrow P$ which classifies the holonomy microbundle over $K_{j}$. 


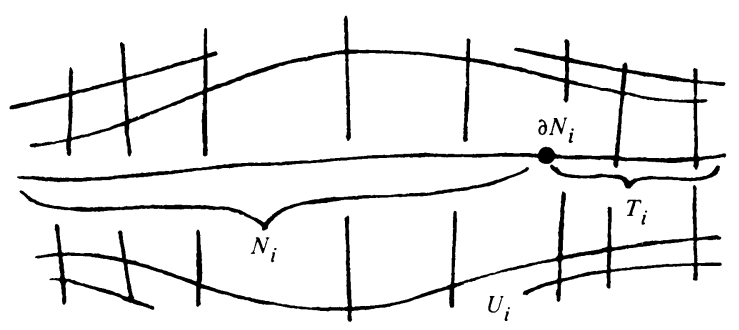

FIGURE 4.1

For each $N_{i}$, let $T_{i}=\partial N_{i} \times(0,1)$ be a collar of $\partial N_{i}$ exterior to $N_{i}$ in $L_{i}$. We may assume (redefining the $U_{i}$ if necessary) that there is an $\mathbf{R}^{n}$-bundle structure $\pi_{i}$ : $U_{i} \rightarrow N_{i} \cup T_{i}$ with fibers transverse to the leaves of $F$ (see Figure 4.1). Then $U_{i}$ is the union of

$$
Y_{i}=U_{i}-\left(N_{i} \cup T_{i}\right) \text { and } Z_{i}=\pi_{i}^{-1}\left(T_{i}\right) \text {, and } Y_{i} \cap Z_{i}=\pi_{i}^{-1}\left(T_{i}\right)-T_{i} .
$$

Recalling that $P$ is a homotopy pushout, we describe $C_{i}: U_{i}-N_{i} \rightarrow P$ by giving maps $\alpha_{i}: Y_{i} \rightarrow G \backslash \mathbf{R}^{n}-0, \beta_{i}: Z_{i} \rightarrow G^{a} \backslash \mathbf{R}^{n}$ and a compatible $\gamma_{i}: \pi_{i}^{-1}\left(T_{i}\right)-T_{i} \rightarrow$ $G^{a} \backslash \mathbf{R}^{n}-0$. The map $\alpha_{i}$ classifies the holonomy microbundle over $N_{i} \cup T_{i}$. Over $T_{i}$, the holonomy microbundle is nonsingular, since $T_{i} \subset M-S$, so we get $\beta_{i}: Z_{i} \rightarrow$ $G^{a} \backslash \mathbf{R}^{n}$, and, by restriction, $\gamma_{i}$.

With proofs of compatibility left to the reader, the map from $M$ to the homotopy pushout $B \Gamma \leftarrow P \rightarrow G \backslash \mathbf{R}$ is defined. To describe the image $R_{k}[M] \in H_{k-1} P$, keep the notation of the preceding discussion. The open sets $U_{i}-N_{i}, V_{j}-K_{j}$ carry fundamental cycles $\left[U_{i}-N_{i}\right] \in H_{k-1}\left(U_{i}-N_{i}\right),\left[V_{j}-K_{j}\right] \in H_{k-1}\left(V_{j}-K_{j}\right)$. Then

$$
R_{k}[M]=\sum C_{i^{*}}\left[U_{i}-N_{i}\right]+\sum C_{j^{*}}\left[V_{j}-K_{j}\right] \in H_{k-1} P .
$$

\section{BiBLIOGRAPHY}

[BK] A. K. Bousfield and D. M. Kan, Homotopy limits, completions and localizations, Lecture Notes in Math., vol. 304, Springer-Verlag, 1972.

[GS] E. Ghys and V. Sergiescu, Sur un groupe remarquable de difféomorphisms du cercle, preprint.

[H1] A. Haefliger, Homotopy and integrability, Manifolds, Amsterdam 1970, Lecture Notes in Math., vol. 197, Springer-Verlag, 1971, pp. 133-163.

[H2] Groupoides d'holonomie et classifants, Asterisque 116, Soc. Math. de France, 1984, pp. $70-97$.

[J] S. Jekel, Loops on the classifying space for foliations, Amer. J. Math. 102 (1980), 13-23.

[M] J. P. May, Simplicial objects in algebraic topology, Van Nostrand, 1967.

[McS] D. McDuff and G. Segal, Homology fibrations and the group completion theorem, Invent. Math. 31 (1976), 279-285.

[Se1] G. Segal, Classifying spaces and spectral sequences, Publ. Math. Inst. Hautes Etudes Sci., No. 34, Paris, 1968, pp. 105-112.

[Se2] , Categories and cohomologv theories, Topology 13 (1974), 293-312.

[Se3] . Classifying spaces related to foliations, Topology 17 (1978), 367-382.

[St] J. Stasheff, Classification via $B \Gamma_{q}$, Lecture Notes in Math., vol. 319, Springer-Verlag, 1973.

[Sa] C.-H. Sah, Automorphism of finite groups, J. Algebra 10 (1968), 47-68.

[T1] R. W. Thomason, Homotopy colimits in the category of small categories, Math. Proc. Cambridge Philos. Soc. 85 (1979), 91-109. 
[Ts] T. Tsuboi, $\Gamma_{1}$-structures avec une seule feuille, Asterisque 116, Soc. Math. de France, 1984, pp. $222-234$.

[T2] R. W. Thomason, First quadrant spectral sequences in algebraic $K$-theory via homotopy colimits, preprint.

[V] R. M. Vogt, Homotopy limits and colimits, Math. Z. 134 (1973), 11-52.

[Ma] J. Mather, Integrability in codimension 1, Comment. Math. Helv. 48 (1973), 195-233.

[E] D. B. A. Epstein, The simplicity of certain groups of homeomorphisms, Compositio Math. 22 (1970), $165-173$.

Departmfent of Mathematics, North Dakota State University, Fargo, North Dakota 58105

Current aàdress: Departamento de Matemáticas, Centro de Investigacion y Estudios Avanzados del IPN, Apartado Postal 14-740, Mexico 14 DF, CP 07000 